\title{
Teaching electric circuits with multiple batteries: A qualitative approach
}

\author{
David P. Smith* and Paul van Kampen ${ }^{\dagger}$ \\ Centre for the Advancement of Science and Mathematics Teaching and Learning, and School of Physical Sciences, \\ Dublin City University, Glasnevin, Dublin 9, Ireland \\ (Received 17 August 2011; published 15 November 2011)
}

\begin{abstract}
We have investigated preservice science teachers' qualitative understanding of circuits consisting of multiple batteries in single and multiple loops using a pretest and post-test method and classroom observations. We found that most students were unable to explain the effects of adding batteries in single and multiple loops, as they tended to use reasoning based on current and resistance where reasoning based on voltage is a necessity. We also found that problems such as thinking of the battery as a source of constant current resurfaced in this new context, and that answers given were inconsistent with current conservation. We describe the curriculum we developed that enables students to model circuits with multiple batteries qualitatively. Post-test results show that the majority of students were able to apply their newly developed model to make accurate predictions for complex circuits.
\end{abstract}

DOI: 10.1103/PhysRevSTPER.7.020115

PACS numbers: 01.40.Fk, 01.40.jc, 01.50.Qb

\section{INTRODUCTION}

In the past three decades, there has been a great deal of research on the teaching and learning of electric circuits, with many different approaches taken [1-5]. At university level, research has mostly concentrated on the qualitative reasoning of preservice science teachers and students in introductory physics courses. The findings of this research have highlighted a number of student misconceptions, including the difficulty of distinguishing between voltage and current. Indeed, many students think that voltage is a property of current [6]. Sometimes, common predictions that are incorrect for voltage hold true for current: for example, von Rhöneck [7] reported that students predict zero voltage across an open switch and a nonzero voltage across a closed switch. Several studies have shown that, even at quite an advanced level, students treat current in purely resistive circuits as the primary concept, while voltage is regarded as a consequence of current and not as its cause [8].

Out of this body of research two main instructional strategies have evolved. Both strategies separate the concepts of voltage, current, and resistance. One deals with voltage as the primary concept, the other, current. Examples of the former approach include Cohen et al. [1], Psillos et al. [3], and Rosenthal and Henderson [5]. Typically, energy transport and (electron) current are distinguished quite early on. Approaches followed by

\footnotetext{
*Present address: Physics Education Group, University of Washington, Department of Physics, Box 351560, Seattle, WA 98195, USA.

†Paul.van.Kampen@dcu.ie

Published by the American Physical Society under the terms of the Creative Commons Attribution 3.0 License. Further distribution of this work must maintain attribution to the author(s) and the published article's title, journal citation, and DOI.
}

McDermott et al. [9] and Tiberghien [10] focus on current first and delay the introduction of voltage.

Other strategies include the comparison of voltage and current to pressure and water flow. The water analogy often goes little deeper than the somewhat ad hoc assertions that "voltage is just like pressure" and "in parallel circuits, current splits into two, just like water does at a fork in a river." However, Schwedes and Dudeck [11] developed curriculum where students develop a complete analogical model for water flow in a closed loop system, using a double water column of constant height difference as a visual analogy for a constant voltage battery, clamped tubes as variable resistors, and water flow meters as light bulbs. Electric circuits are then introduced in analogy with the water loop model.

Although all approaches deal with the concept of voltage in some detail, they do not put much emphasis on what happens to circuits when the number of batteries is increased. Yet, there is some important elementary physics to be gleaned from considering such circuits, as they may serve to introduce more advanced concepts such as potential (as distinct from potential difference) in a straightforward manner [12]. Curriculum on multiple batteries also affords students another opportunity to differentiate between current and voltage. In this paper, we discuss curriculum on the addition of batteries in various ways that achieves both of these goals and leads naturally to a qualitative treatment of Kirchhoff's loop rule in multipleloop circuits.

While most introductory university physics textbooks provide a quantitative treatment of Kirchhoff's rules, the development of a coherent conceptual framework that allows students to consider multiple-loop multiple-battery circuits without recourse to solving equations is typically not a goal of instruction. Often, circuits are given with a number of linear resistors and batteries configured in a 
small number of loops, like the circuit of Fig. 1(a). Students are encouraged to label the currents through all loops, assume a direction, and apply Kirchhoff's loop and junction rules. Many of the circuits that appear resemble those selected by Woodman [13] and allow for a straightforward stepwise solution of the many equations with many unknowns that emerge. Nevertheless, the emphasis is on developing techniques to find a numerical solution rather than on developing a conceptual framework based on qualitative reasoning. For example, textbooks can seem to encourage students to randomly assume directions for current at different junctions, and if the sign of a current comes out negative, this is typically shrugged off as inconsequential- "we just assumed the wrong direction." (This is of course different from situations where students through qualitative reasoning predict the direction of the currents, in which case a negative sign provides a valuable numerical check on previous reasoning.)

When dealing with linear resistors only, the absence of building a conceptual framework could be seen as no worse than an opportunity missed. However, circuits containing nonlinear resistors such as bulbs cannot be treated in this way. For example, to analyze the elementary circuits of Figs. 1(b) and 1(c) a qualitative approach becomes a necessity. To illustrate the point, the currents in the circuit of Fig. 1(a) are readily found to be $i_{1}=V_{0} / R_{1}, i_{2}=V_{0} / R_{2}$, and $i_{3}=V_{0}\left(1 / R_{1}-1 / R_{2}\right)$. Given constant values for $V_{0}$, $R_{1}$, and $R_{2}$, straightforward substitution will yield numerical values for the currents. When $R_{1}=R_{1}\left(i_{1}\right)$ and $R_{2}=$ $R_{2}\left(i_{2}\right)$, as is the case for bulbs, values for the currents and resistances can be found through an iterative procedure, but only if the $i, V$ characteristic of the bulb is known. Therefore, if the non-Ohmic behavior (i.e., currentdependent resistance) of bulbs is to be taken into account, a qualitative approach becomes a practical necessity.

In this paper, we describe a research-validated approach to the teaching and learning of circuits containing multiple batteries and bulbs in multiple loops. In Sec. II, we describe the prerequisite knowledge and understanding of simpler electric circuits and the prior exposure of our students. In Sec. III, we describe student difficulties with multiple batteries in single loops, the curriculum developed to address these, and post-test results. Section IV details pretest results, curriculum, and post-test results for multiple batteries in multiple loops.
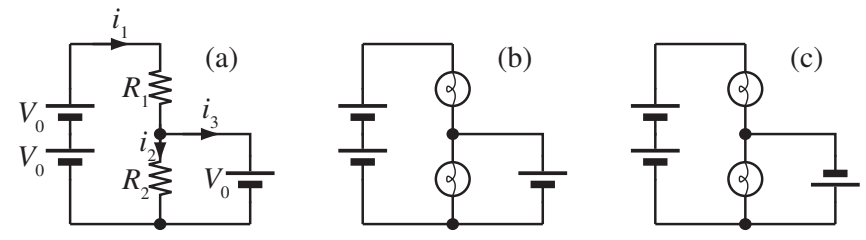

FIG. 1. Circuits with multiple batteries in multiple loops. Standard methods can be used to solve problems with linear resistors like (a), but fail to deal with nonlinear resistors as in circuits (b) and (c).

\section{PREREQUISITES}

The curriculum we developed was used to supplement parts A-C of the Electric Circuits curriculum in Ref. [9]. It has been used in courses for preservice and in-service teachers at Dublin City University (DCU), the University of Washington, and the University of Maine. At DCU the groups were sufficiently large that meaningful pre- and post-test data could be obtained. Typically, the pre- and post-test questions were answered by 30-50 students, which is not a large enough cohort to allow for a robust quantitative analysis. Nevertheless, we think it is meaningful to indicate the prevalence of certain answers in percentages rounded to the nearest 5\%; however, one must bear in mind that the error bars are larger than suggested by this practice.

The curriculum on multiple batteries in multiple loops should be usable as a stand-alone provided the students' model for electric circuits incorporates the following elements:

(1) There is a current in complete circuits. This current is not used up.

(2) The magnitude of the current through a battery depends on the resistance of the circuit. When the resistance of the circuit increases, the current through the battery decreases, and when the resistance of the circuit decreases, the current through the battery increases.

(3) Kirchhoff's junction rule, including the rule that in a single-loop circuit the current is the same throughout.

(4) The voltage across the terminals of a battery is constant. (In practice, even when low-resistance bulbs are used with $1.5 \mathrm{~V} \mathrm{D}$ batteries, the deviation from ideal behavior is negligibly small.)

(5) Kirchhoff's loop rule applied to single-battery circuits.

(6) The brightness of a bulb increases when the current through it increases. The brightness of a bulb also increases when the voltage across it increases. Thus, bulb brightness links current and voltage qualitatively. Ohm's law does not apply to bulbs.

Another useful skill, further developed in these labs, is the ability to transfer between circuit diagrams and physical circuits in the laboratory.

As we will demonstrate in Secs. III and IV, even students who have a good grasp of these concepts (as evidenced by post-testing) typically do not correctly generalize these to circuits with multiple batteries.

\section{MULTIPLE BATTERIES IN A SINGLE LOOP}

In this section, we discuss common student difficulties we identified in relation to circuits containing more than one battery. The batteries are connected either in series or in parallel with each other (but not in parallel with other 

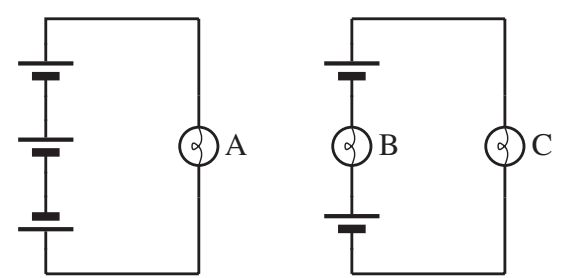

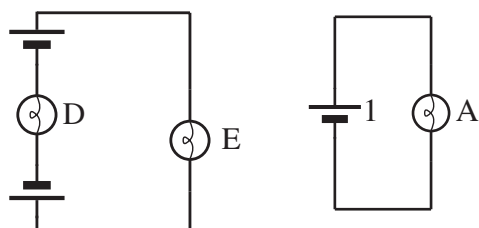

(a)

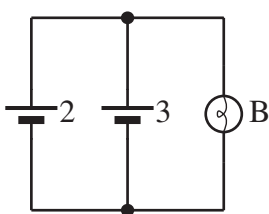

(b)

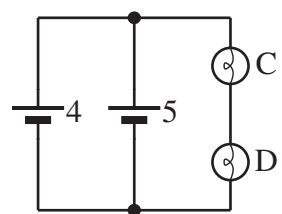

(c)

FIG. 2. Circuit diagrams used in multiple batteries in series pretest. Students are asked to rank the bulbs by brightness, and explain the reasoning behind their ranking.

circuit elements). For brevity, we say that these circuits contain multiple batteries in a single loop. We also discuss the curriculum we developed to address these difficulties and analyze post-test results.

\section{A. Multiple batteries in series-Pretest analysis}

Before we administered the curriculum, we gave students a pretest question pertaining to the circuits of Fig. 2. The question examines student understanding of how the addition of batteries in series in different orientations affects bulb brightness. It also implicitly addresses understanding of series circuits in a new context.

Only 3 students out of 39 , i.e., about $10 \%$, ranked the bulbs correctly by brightness $(\mathrm{A}=\mathrm{B}=\mathrm{C}>\mathrm{D}=\mathrm{E})$. The most prevalent incorrect answers are listed in Table I. Some $55 \%$ of students state that bulb A will not light. Without exception, they reasoned that the battery in opposite orientation "blocks the current" or "must have a really high resistance." In many cases this reasoning appears to stem from experiences with putting batteries "the wrong way" into household appliances. However, this reasoning was not applied in a way that is internally consistent, or consistent with the model described in Sec. II, as smaller fractions of students predicted that bulb D (50\%) and bulb E (35\%) will not light.

It is also worth noting that $10 \%$ of students rank bulb C brighter than bulb $\mathrm{B}$, or bulb $\mathrm{E}$ brighter than bulb $\mathrm{D}$. Analysis of students' answers reveals that it is not the case that the old misconception of current being used up resurfaces; instead, these students reason that the batteries supply different amounts of current to different bulbs or that there can be no current flow between two negative terminals. In doing so, they appear to revert to ideas of

TABLE I. Common incorrect answers to multiple batteries in series pretest question of Fig. 2.

\begin{tabular}{lc}
\hline \hline Incorrect answer & Pretest $(N=39)$ \\
\hline Bulb A will not light & $55 \%(21)$ \\
Bulb D will not light & $50 \%(19)$ \\
Bulb E will not light & $35 \%(14)$ \\
Bulb C is brighter than bulb B & $10 \%(3)$ \\
Bulb E is brighter than bulb D & $10 \%(3)$ \\
\hline \hline
\end{tabular}

FIG. 3. Circuit diagrams used in multiple batteries in parallel pretest. Students are asked to rank the bulbs by brightness and the batteries by current, and explain their ranking.

batteries supplying current to empty wires and the current stopping and starting at the battery terminals, and abandon the notion that the current is the same at every point in a single loop.

\section{B. Multiple batteries in parallel-Pretest analysis}

To test students' initial ideas on batteries connected in parallel with each other, they were asked to rank the brightness of the bulbs and the currents through the batteries in circuits (a)-(c) of Fig. 3. The question also allows us to probe to what extent the prior instruction described in Sec. II had been successful.

Table II summarizes the answers given. Of the six students $(20 \%, N=27)$ who ranked the bulbs correctly $(\mathrm{A}=\mathrm{B}>\mathrm{C}=\mathrm{D})$, three did not provide a reason for why they ranked bulbs A and B brightest, and two others merely stated that the circuits of Figs. 3(a) and 3(b) contained just one bulb. Only one student explicitly gave correct reasoning, based on voltage.

It is more instructive to consider partial rankings. We found that 25 students (95\%) correctly ranked bulbs $\mathrm{C}$ and $\mathrm{D}$ as being equally bright with correct reasoning. Likewise, 95\% ranked bulb B brighter than $\mathrm{C}$, with correct reasoning. With just one exception, all reasoning was based on current and resistance, and not on voltage. We regard these results as evidence that the prior instruction described in Sec. II has been successful.

Seventeen students (65\%) ranked bulb B brighter than bulb $\mathrm{A}$. One of these students based her reasoning on voltage; she thought the voltage across bulb $\mathrm{B}$ would be twice that across bulb A. The other 16 based their reasoning on the idea that each battery supplies a current to the circuit;

TABLE II. Common rankings for multiple batteries in parallel pretest question of Fig. 3. In all tables, bold font indicates correct answers.

\begin{tabular}{lccc}
\hline \hline Battery ranking & $(N=27)$ & Bulb ranking & $(N=27)$ \\
\hline $\mathbf{1}>\mathbf{2}=\mathbf{3}>\mathbf{4}=\mathbf{5}$ & $\mathbf{1 0 \%}(\mathbf{3})$ & $\mathbf{A}=\mathbf{B}>\mathbf{C}=\mathbf{D}$ & $\mathbf{2 0 \%}(\mathbf{6})$ \\
$2=3>4=5>1$ & $15 \%(4)$ & $\mathrm{B}>\mathrm{A}>\mathrm{C}=\mathrm{D}$ & $25 \%(7)$ \\
Partial rankings: & & $\mathrm{B}>\mathrm{A}=\mathrm{C}=\mathrm{D}$ & $20 \%(5)$ \\
$\mathbf{2}=\mathbf{3}, \mathbf{4}=\mathbf{5}$ & $\mathbf{5 0 \%}(\mathbf{1 3})$ & Other & $35 \%(9)$ \\
$2<3,4<5$ & $20 \%(6)$ & & \\
$2>3,4>5$ & $10 \%(3)$ & & \\
\hline \hline
\end{tabular}


it appears that they implicitly assumed that this current is independent of the circuit. In classroom discussions some of these students introduce ideas akin to superposition. This assumption leads them to believe that bulb B is brighter than bulb A since it is supplied current from two batteries rather than one. This generalization satisfies the junction rule but is inconsistent with reasoning based on voltage (either via the loop rule or observed bulb brightness). The three students who thought bulb A would be brighter than bulb B did not explain their reasoning, and one student's answer did not rank bulb A relative to bulb B. Our findings here are similar to those documented by Licht [14].

The 27 students gave 18 different rankings for the current through the batteries. The six students who correctly ranked the brightness of the bulbs in Fig. 3 $(\mathrm{A}=\mathrm{B}>\mathrm{C}=\mathrm{D})$ gave six different rankings for the current through the batteries; none were consistent with their bulb rankings. When these students were trying to make sense of the unfamiliar circuits, they did not treat the junction rule as inviolable.

Just under half of the students (13 out of 27) correctly stated that the currents through batteries 2 and 3 on the one hand, and 4 and 5 on the other hand, would be equal. They appeared to use symmetry reasoning. Six out of 27 , or $20 \%$, thought that the currents through the batteries drawn closest to the bulbs would be greater. Their reasoning tended not to be sufficiently detailed to draw any firm conclusions, but it could be the reappearance of an old notion some of them may have had: that proximity to the battery matters. Indeed, a pretest question posed much earlier in the course concerned a circuit like that of Fig. 3(b), but with battery 3 replaced with a bulb. In that situation, about $25 \%$ of students reckoned that this bulb will get more current than bulb B because of its closer proximity to battery 2 .

Finally, 10\% ranked the current through battery 2 greater than that through battery 3 , and the current through battery 4 as greater than that through 5. One student wrote, this is because the current through these batteries [i.e., 3 and 5] experiences no resistance. This student focuses on one aspect that was important when adding bulbs in parallel, but in doing so apparently treats the "original" battery as different from the battery that was "added."

In summary, we have found that students have a number of misconceptions when dealing with circuits containing multiple batteries in a single loop. Specifically, a large number of students believe that bulbs in circuits with batteries in opposite orientation will not light. They either state that the battery in opposite orientation does not permit current in these circuits or that the circuits are incomplete. It is also evident that old notions, such as the battery supplying a (constant) current, resurface in this new context.

Additionally, we find that students tend to base their answers primarily on current and resistance when analyzing these types of circuits. This cannot be termed a misconception, but it suggests a lack of understanding and confidence in conceptual reasoning based on voltage.

\section{Curriculum}

To appreciate the pretest questions asked on circuits with multiple batteries in multiple loops, we describe the curriculum developed to address the difficulties identified in the pretests on multiple batteries in single loops here. Students start by investigating the addition of batteries in series (in the same orientation) in four different elementary circuits: a single-bulb circuit, a two-bulb series circuit, a two-bulb parallel circuit, and one with three bulbs, two of which are in parallel. The students devise a rule like: When I add a second battery in series with another battery (in the same orientation), the brightness of all bulbs in the circuit increases. Students then investigate how adding batteries in parallel affects bulb brightness, allowing them to form another rule: When I add a second battery in parallel with another battery (in the same orientation), the brightness of all bulbs in the circuit remains the same. Students then carry out three more experiments in which they add batteries in opposite orientation in single-loop circuits. They discover that when a battery is added in opposite orientation, it effectively cancels a battery already connected in the circuit, and that the order in which batteries and bulbs are connected does not affect bulb brightness or the current in the circuit. A small number of structured experiments and exercises suffices here for almost all students; they find it easy to incorporate the new rules into their model.

The focus of this part of the curriculum is on current and bulb brightness, not on voltage. If this part of the curriculum were used without the multiple-loops curriculum described in Sec. IV, we would add a number of exercises that explicitly make students think of the effect of adding batteries in terms of voltage. We would not envisage many problems for students if this approach were adopted.

\section{Multiple batteries in a single loop-Post-test analysis}

We post-tested the effect of adding batteries in series in different orientations as part of a long sequence of questions that probed other aspects of the curriculum at the same time, either by adding an extra battery or reversing its orientation. Over $90 \%$ of answers were correct in each case.

A post-test question on the addition of batteries in parallel with other batteries, shown in Fig. 4, was given during three years, with slight variations in the ordering and labeling of batteries. The questions are directly comparable, which gives us a pool of 50 answers to work with. Here batteries are connected both in parallel with each other and in series, a configuration not encountered in the curriculum.

In the first question, we asked students to rank all circuit elements by current when both switches are open. As Table III shows, just over half the students answered the 


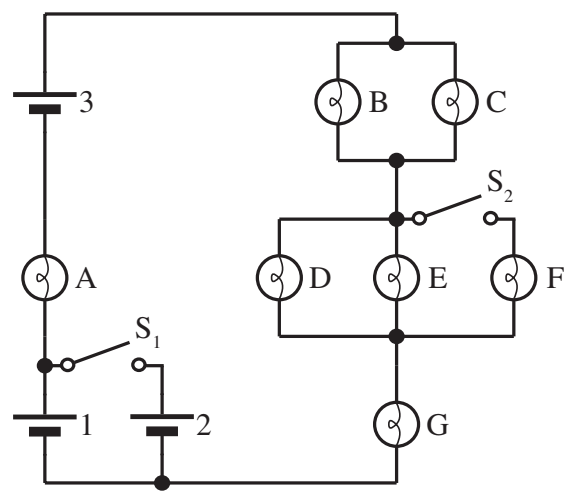

FIG. 4. Circuit diagram used in the multiple batteries in a single loop post-test.

question completely and correctly, while another $10 \%$ ranked the batteries and bulbs correctly but separately. A further $10 \%$ did not rank the batteries at all; the remaining $30 \%$ gave different answers that are difficult to categorize.

In the second question, students were asked to state what happens to the brightness of bulb D when switch 1 is closed. More than $60 \%$ correctly stated that its brightness would not change, and about one-third stated that it would increase. This is a significant improvement on the corresponding pretest data (20\% stated that bulbs A and B would be equally bright; see Table II). However, only $20 \%$ ranked the currents through all circuit elements correctly in this case. Another 20\% thought that battery 2 would get no current; this answer is consistent with the fact that the circuit appears to be unaffected, but does not incorporate the symmetry arguments discussed in the curriculum. Fifteen percent made an incorrect generalization and thought that all three batteries would receive equal currents. Surprisingly, 10\% stated that the current would split in two equal proportions between batteries 1 and 2 as well as between bulbs $\mathrm{B}$ and $\mathrm{C}$ and bulbs $\mathrm{D}$ and $\mathrm{E}$, but nevertheless ranked the currents through the parallel

TABLE III. Bulb rankings in multiple batteries in single loops post-test of Fig. 4.

\begin{tabular}{ll}
\hline \hline Ranking & Total $(N=50)$ \\
\hline When switch 1 is open: & \\
$\mathbf{1}=\mathbf{3}=\mathbf{A}=\mathbf{G}>\mathbf{B}=\mathbf{C}=\mathbf{D}=\mathbf{E}>\mathbf{2}=\mathbf{F}=\mathbf{0}$ & $\mathbf{5 0 \%}(\mathbf{2 6})$ \\
Batteries and bulbs ranked separately but correctly & $10 \%(6)$ \\
Batteries not ranked, bulbs ranked correctly & $10 \%(4)$ \\
Other & $30 \%(14)$ \\
When switch 1 is closed: & \\
Brightness of bulb $\mathbf{D}$ is unchanged & $\mathbf{6 0 \%}(\mathbf{3 1})$ \\
Bulb $\mathrm{D}$ gets brighter & $35 \%(17)$ \\
Bulb $\mathrm{D}$ gets dimmer & $<5 \%(2)$ \\
$\mathbf{3}=\mathbf{A}=\mathbf{G}>\mathbf{1}=\mathbf{2}=\mathbf{B}=\mathbf{C}=\mathbf{D}=\mathbf{E}>\mathbf{F}=\mathbf{0}$ & $\mathbf{2 0 \%}(\mathbf{9})$ \\
$1=3=\mathrm{A}=\mathrm{G}>\mathrm{B}=\mathrm{C}=\mathrm{D}=\mathrm{E}>2=\mathrm{F}=0$ & $20 \%(9)$ \\
$3=\mathrm{A}=\mathrm{G}>1=2>\mathrm{B}=\mathrm{C}=\mathrm{D}=\mathrm{E}>\mathrm{F}=0$ & $10 \%(6)$ \\
The current through all batteries is equal & $15 \%(7)$ \\
Other & $40 \%(19)$ \\
\hline \hline
\end{tabular}

batteries greater than the currents through the parallel bulbs. This may be an artifact of how the question was posed, but we really cannot explain this to our own satisfaction, as none of these students explicitly stated why the bulb and battery currents would be different.

\section{MULTIPLE BATTERIES IN MULTIPLE LOOPS}

\section{A. Batteries in parallel with other networks-Pretest analysis}

To investigate how students deal with multiple batteries in multiple loops (i.e., batteries that are connected with each other neither in series nor in parallel), we asked them to rank the bulbs in the circuits of Fig. 5 by brightness. To answer the question completely, students must reason both in terms of current and voltage.

The most prevalent answers are listed in Table IV. All students appeared to consider the three-battery circuit in terms of the difference with the two-battery circuit (i.e., the addition of an extra battery); none seemed to look at the circuit as consisting of two parallel branches (one consisting of bulb D and one consisting of batteries 3 and 4 and bulb C) connected to battery 5 .

Even though $35 \%$ of students ranked the bulbs correctly, none provided correct reasoning. Eleven of these 13 students reasoned that the addition of a battery in parallel does not affect the brightness of bulbs in a circuit. Most of these students based their reasoning on their observations that connecting a battery in parallel to a single-battery, singlebulb circuit did not affect bulb brightness, and have incorrectly generalized this rule.

Eight students stated that bulb $\mathrm{C}$ would be brighter than bulbs A and B (which have the same brightness), and that these are brighter than bulb D. They treated battery 5 as a circuit element with characteristics varying from a wire to a bulb, with otherwise correct reasoning, for example:

All the current through the two batteries goes through $C$. $A$ and $B$ are arranged in series so voltage across them is halved between them. Their brightness is less than C. D is in parallel with battery 5. This branch has little resistance and the current will go through battery 5 and $D$ will be shorted out.

As there is less resistance due to [battery 5] connected in parallel across bulb $D$ it has less voltage across it than bulb $C$ and is therefore less bright than bulb $C$.
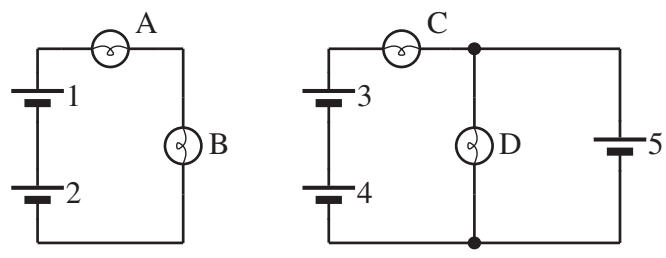

FIG. 5. Circuit diagrams used in the multiple batteries in multiple loops pretest. Students are asked to rank the bulbs by brightness. 
TABLE IV. Bulb rankings in the multiple batteries in multiple loops pretest of Fig. 5.

\begin{tabular}{lc}
\hline \hline Ranking & Total $(N=40)$ \\
\hline $\mathbf{A}=\mathbf{B}=\mathbf{C}=\mathbf{D}$ & $\mathbf{3 5 \% ( 1 3 )}$ \\
$\mathrm{C}>\mathrm{A}=\mathrm{B}>\mathrm{D}$ & $20 \%(8)$ \\
$\mathrm{A}=\mathrm{B}>\mathrm{C}=\mathrm{D}$ & $15 \%(6)$ \\
$\mathrm{D}>\mathrm{A}=\mathrm{B}=\mathrm{C}$ & $10 \%(4)$ \\
$\mathrm{A}=\mathrm{B}=\mathrm{C}>\mathrm{D}$ & $10 \%(3)$ \\
Other & $15 \%(6)$ \\
\hline \hline
\end{tabular}

While it is in itself encouraging that students make comparisons to other circuit elements, and then reason consistently, we also note that student reasoning is still predominantly based on current and resistance. However, voltage does begin to make more of an appearance, even though it does not appear to have been fully incorporated into their model.

Many others appeared to treat the battery as a source of current. For example, four students reasoned that bulb D would be brightest, since it received current from three batteries:

$D>C=A=B$. Bulb $D$ receives the current through the 2 batteries in series and also the one in parallel.

Six students thought that bulbs A and B would be equally bright, and bulbs $\mathrm{C}$ and $\mathrm{D}$ would be equally bright but dimmer than bulbs $\mathrm{A}$ and $\mathrm{B}$, by reasoning that battery 5 opposes batteries 3 and 4 . This may be the result of incorrectly generalizing from their experience with multiple batteries in single loops.

\section{B. Curriculum-Electric potential}

To help address the issues identified in the pretest, we introduced the concept of potential in the by-now familiar context of single-loop circuits. In a first version of the curriculum, we only introduced potential when it was essential (see Sec. IVC). However, introducing it in the single-loop curriculum makes it easier for students to link potential to circuits containing multiple batteries in single loops, which they understand well in terms of current and resistance. Thus, it both completes the prior curriculum and bridges it with the more complex circuits that are to follow.

Students use the circuits of Fig. 6 to find that all voltages across individual circuit elements can be deduced from knowing the potential differences between one of the points (say, $A$ ) and all others. We then introduce the concept of electric potential (at any point $P$ ) by defining it as the potential difference between points $P$ and $A$. The students arrive at the following key conclusions:

- the potential at the positive terminal of a battery is greater than the potential at the negative terminal of the same battery by an amount equal to the battery voltage;

- the potential across a bulb changes by an amount equal to the voltage across the bulb;
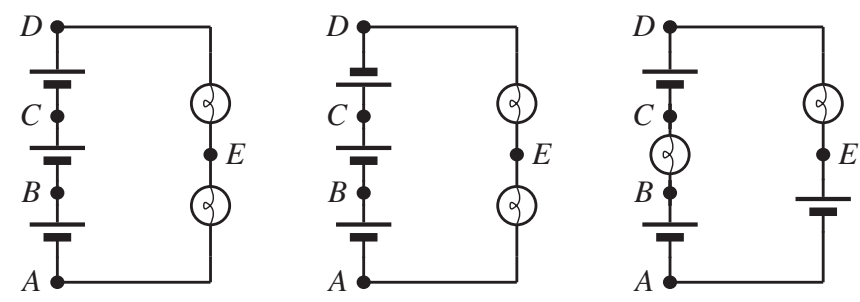

FIG. 6. Circuit diagrams used to develop the idea of potential. Students note the voltages across different elements and the orientation of the voltmeter leads.

- the current through a bulb is always in the direction from high potential to low potential;

- the current through a battery can be in either direction.

\section{Curriculum-Multiple batteries in multiple loops}

The curriculum described in Sec. IV B contains all the rules the students need to predict and explain the behavior of many multiple-loop circuits qualitatively. To give an example, it is a significant challenge to enable students to understand that, and analyze how, the two circuits shown in Fig. 7 behave differently. The concept of potential helps them achieve this.

In the Physics by Inquiry curriculum [9], students have seen that, if the switch is closed in the circuit of Fig. 7(a), the parallel bulb A is not affected (in that case, the box represents any arrangement of bulbs, excluding those that would cause short-circuiting). In the circuit of Fig. 7(b), bulbs $\mathrm{B}$ and $\mathrm{C}$ do change in brightness when the switch is closed.

Students find that these rules still hold when the boxes are allowed to contain batteries, with some caveats. For example, in the circuit of Fig. 7(a), the batteries inside the box must not be arranged in such a way that bulb A is effectively short-circuited (for example, if the box contains just one battery). Likewise, the brightness of bulb B could adventitiously remain unchanged if the box contains a single battery.

Regardless of whether the curriculum is used with or without prior exposure to the Physics by Inquiry curriculum [9], the distinction between the two kinds of circuits

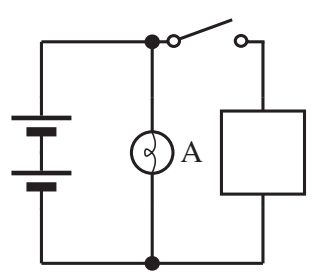

(a)

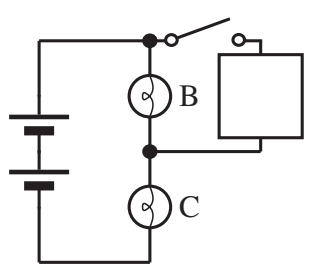

(b)
FIG. 7. Parallel connections (a) directly across the battery, (b) in series with another circuit element. The boxes represent any arrangement of bulbs and batteries. 


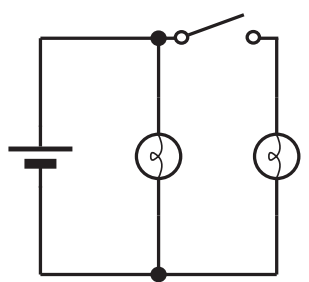

(a)

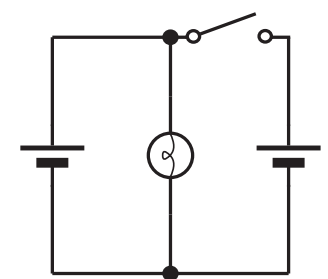

(b)
FIG. 8. Circuits used to highlight the similarities and differences between connecting in parallel with a bulb and battery (a) a second bulb and (b) a second battery.

helps to convey that a battery maintains a constant voltage between its terminals. Of course this observation is valuable in its own right, but it also appears to help students accept at a visceral level that the current through identical batteries depends on how they are connected.

To get students thinking about current through and voltage across a battery, they revisit the two circuits given in Fig. 8. From the unchanged bulb brightness, they infer that on closing the switch the voltage across the battery and bulb remains constant. In the circuit of Fig. 8(a) the current through the battery must double, but in that of Fig. 8(b) the current through the original battery halves (by symmetry) [15]. Students also consider that if they just looked at the outer loop of the latter circuit, they might conclude that there is no current in the circuit at all. Thus, early on students are encouraged to take a view of the circuit as a system, and not to apply reasoning that is sound for one loop incorrectly to a different loop.

The main body of the curriculum revolves around the circuit of Fig. 9(a). The students already have all the tools necessary to predict what will happen when the switch

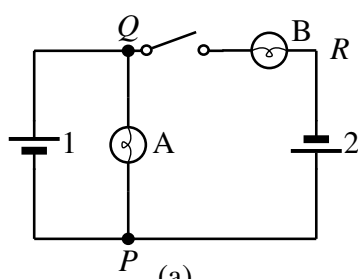

(a)

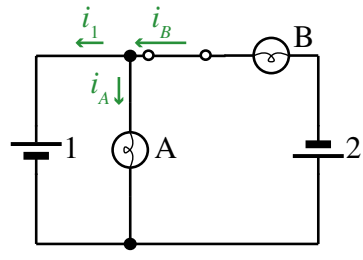

(c)

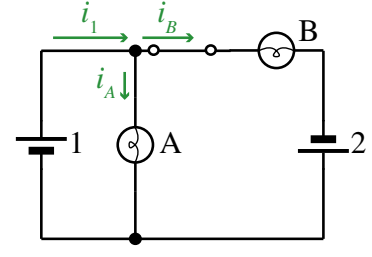

(b)

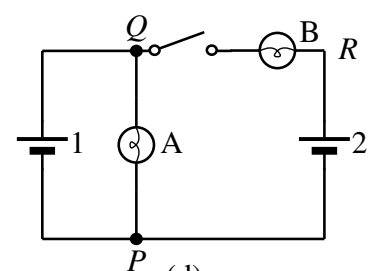

(d)
FIG. 9 (color online). Two circuits with multiple batteries in multiple loops used to develop the concept of potential and determine the direction of current. Circuits (a)-(c) are identical; in circuits (b) and (c), the length of the arrow indicates the magnitude of the current. closes, but many are surprised at their observations when they verify their predictions. Students typically have not applied their model for potential described in Sec. IV B spontaneously; but when explicitly asked to do so, they readily find that $V(R)+2 V_{0}=V(P)+V_{0}=V(Q)$, where $V_{0}$ is the battery voltage. From this they deduce that bulb B is brighter than bulb A, and that the direction of current is from $Q$ to $R$, and from $Q$ to $P$. By applying Kirchhoff's junction rule, students infer that all current flows through battery 1 , splits at $Q$, and recombines at $P$. Note that without the rules for current through a bulb described in Sec. IV B, students could come up with four directions for the current to explain the observed relative brightness of bulbs A and B. Two of these are given in Fig. 9(b) (correct) and Fig. 9(c) (incorrect); the other two possibilities have all three currents reversed.

Students next encounter the circuit of Fig. 9(d). They now find that $V(R)=V(Q)=V(P)+V_{0}$, and hence that there is no current through bulb B and battery 2 . The difference in behavior of the two circuits of Figs. 9(a)-9(d) is very striking and memorable. To embed these new ideas more firmly, students then investigate three more circuits (not shown in this paper) where a third battery has been added in series with battery 2 .

\section{Post-test analysis}

We present data on two post-test questions examining multiple batteries in multiple loops. One concerned a circuit quite similar to the circuits presented in the curriculum, the other was quite different so that we could probe the depth of students' understanding further.

The first post-test question under discussion asked students $(N=31)$ to rank the relative brightness of the bulbs in the circuit shown in Fig. 10. Table V reveals that almost all students (95\%) obtained the correct answer; just over half of those used similar reasoning to that outlined in Sec. IV C, while a further $25 \%$ very likely did so but did not make all steps explicit (for example, they gave consistent values for potential at various points and stated that the voltage across bulbs $\mathrm{C}$ and $\mathrm{D}$ would be greater than the voltage across bulbs $\mathrm{A}$ and $\mathrm{B}$, without explaining why). Twenty percent used reasoning based on application of Kirchhoff's loop rule to just one or two loops. These

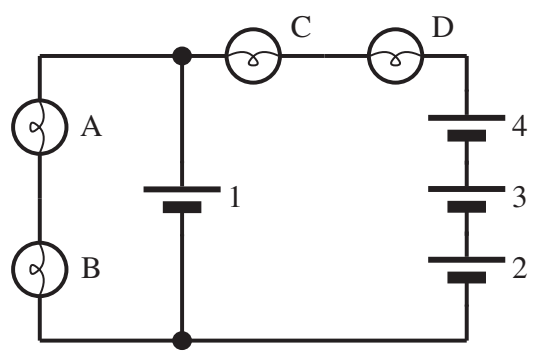

FIG. 10. Circuit diagram used in a multiple batteries in multiple loops post-test. 
TABLE V. Bulb rankings in the multiple batteries in multiple loops post-test of Fig. 10.

\begin{tabular}{lc}
\hline \hline Ranking & Total $(N=31)$ \\
\hline $\mathbf{C}=\mathbf{D}>\mathbf{A}=\mathbf{B}$ & $\mathbf{9 5 \%}(\mathbf{2 9})$ \\
Complete correct reasoning & $\mathbf{5 5 \% ( 1 7 )}$ \\
Correct but incomplete reasoning & $25 \%(7)$ \\
Correct but incorrect reasoning & $15 \%(5)$ \\
$\mathrm{A}=\mathrm{B}=\mathrm{C}=\mathrm{D}$ & $5 \%(2)$ \\
Upward current through batteries $\mathbf{2 - 4}$ & $\mathbf{9 0 \%}(\mathbf{2 8})$ \\
Downward current through battery $\mathbf{1}$ & $\mathbf{6 5 \%}(\mathbf{2 0})$ \\
Upward current through battery 1 & $30 \%(10)$ \\
\hline \hline
\end{tabular}

students concluded that, based on the left loop, the voltage across bulbs A and B was half the voltage across a battery, while based on the right loop, the voltage across bulbs $\mathrm{C}$ and $\mathrm{D}$ was twice the voltage across a battery. Thus they obtained an answer that is qualitatively correct but quantitatively in error.

In the second part of the question, students were asked to indicate the direction of current through the batteries. To correctly reason the direction of current through batteries $2-4$, students should refer to the direction of current through bulbs $\mathrm{C}$ and D. On this basis, the current through batteries 2-4 is upwards. To determine the direction of current through battery 1 , students must reason on the basis of potential while satisfying Kirchhoff's junction rule. This allows them to determine that the direction of current through bulbs $\mathrm{C}$ and $\mathrm{D}$ is from right to left and the direction of current through bulbs A and B is downwards. Since the voltage across bulbs $\mathrm{A}$ and $\mathrm{B}$ is half the voltage across bulbs $\mathrm{C}$ and $\mathrm{D}$, they are dimmer, less current flows through them, and hence by Kirchhoff's junction rule the current through battery 1 must flow downward.

The direction of the current through batteries 2-4 was determined correctly by $90 \%$ of the students, but only $25 \%$ used the reasoning based on the direction of current through bulbs $\mathrm{C}$ and $\mathrm{D}$. The majority of the remaining answers merely stated the convention that the current through the battery is from the negative terminal to the positive terminal inside a battery. However, this appears to give too pessimistic a picture: if this were the sole basis for their reasoning, we would expect all 20 of these students to state that the current through battery 1 would also be upwards, but only half of them (10) did. In all, 20 students correctly stated that the current through battery 1 would be in the downwards direction, with eight providing complete reasoning and another eight providing incomplete reasoning. Four of the correct answers were based on partially incorrect reasoning.

The second post-test question we consider concerns the circuit shown in Fig. 11. Note that this question was given to only 14 students, so the statistics are only indicative. However, we include it here to show to what extent transfer to different types of circuits takes place. The circuit is quite

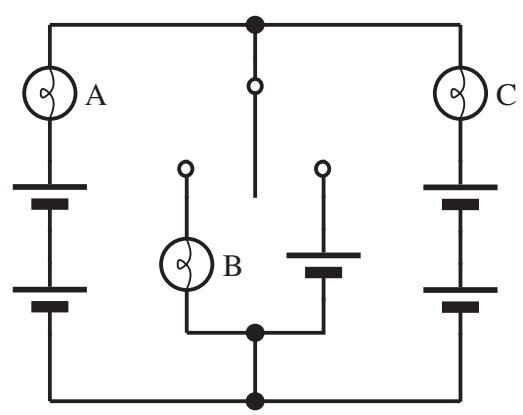

FIG. 11. Circuit diagram used in a multiple batteries in multiple loops post-test.

different from what the students had encountered in the curriculum in that there are two branches with a bulb and a battery; not only does it look different, to obtain an answer Kirchhoff's junction rule must be applied before the rules on potential.

In the first part of the question, students were asked to rank the relative brightness of the bulbs when the switch was in the center position. Some $65 \%$ of students correctly stated that none of the bulbs would light when the switch was in the center position as shown above; all used correct and complete reasoning. The remaining students noted that bulbs $\mathrm{A}$ and $\mathrm{C}$ were connected in series but failed to take into account that the batteries effectively cancel each other; they stated that bulbs A and C would be equally bright (but not off).

In the second question of the post-test, students were once again asked to rank the brightness of bulbs when the switch in the circuit of Fig. 11 was turned to the right, adding a battery to the circuit. While $70 \%$ of the students gave a correct ranking, only half of these gave complete and correct reasoning; $30 \%$ gave correct but incomplete reasoning, while $20 \%$ were unclear or incorrect.

In the last question, students were asked to rank the brightness of the bulbs when the switch in the circuit of Fig. 11 was moved to the left. Reasoning here is a little more intricate; algorithmically considering potentials first and then applying the junction rule will not work. Students should consider that the potential below bulb B is less than the potential above bulb B, so current is in the downward direction. By symmetry, the currents through bulbs A and $\mathrm{C}$ are equal and in the same direction; by Kirchhoff's junction rule, these currents must be upward. Thus bulb $\mathrm{B}$ must be brighter than bulbs $\mathrm{A}$ and $\mathrm{C}$, which are equally bright.

No student used this line of reasoning, although three students did provide the correct ranking with incomplete reasoning. All three used reasoning based on voltage; they all stated that the voltage across bulb B was greatest, but did not give a complete answer, for example:

Bulb $B$ will be the brightest. Parallel branches have equal voltages across them and the branches containing bulbs $A$ and $C$ have batteries on them. if the sum of the 
voltages of each element on a branch is equal to the sums of the $V$ of all of the elements on the next branch then $B$ will be brightest as it is on a branch on its own. The other 2 branches have batteries on them which have a voltage i.e., $V_{1}+V_{2}+V_{A}=V_{B}$ and $V_{3}+V_{4}+V_{C}=V_{B}$. [...] the voltages of $\mathrm{A}+\mathrm{C}$ are equal + they will be the same brightness.

\section{CONCLUSION}

This research has extended the body of physics education research on students' understanding of circuits consisting of multiple batteries in multiple loops. Pretest analysis identified that a large majority of students are unable to explain the effects of adding batteries in single and multiple loops. In answering these questions, students relied mostly on their understanding of current and resistance, while reasoning based on voltage is a necessity. Even though the belief that the battery supplies current, albeit a variable current, to "empty" wires and bulbs resurfaced, some students did display an ability to model a circuit based on their conceptual understanding of voltage, current, and resistance.
The introduction of the concept of potential allowed students to systematically discover rules for how circuits with multiple batteries in multiple loops can be modeled. Post-test results show that the majority of students were able to apply their newly developed model to make accurate predictions for complex circuits. The analysis of their answers also revealed an increased understanding of the roles current, voltage, and resistance play in their model for electric circuits. However, students still found it difficult to transfer their understanding to a new context.

\section{ACKNOWLEDGMENTS}

We gratefully acknowledge fruitful discussions with Thomas Wemyss, John Thompson, Peter Shaffer, and MacKenzie Stetzer. We are also grateful to Roger Feeley and Benjamin Pratt for using the curriculum in their institutions and for their very useful feedback, and to Enda McGlynn and Mila Kryjevskaia for a thorough reading of the manuscript. This work was supported by the Irish Research Council for Science Engineering and Technology, Dublin City University's Learning Innovation Unit and the School of Physical Sciences.
[1] R. Cohen, B. Eylon, and U. Ganiel, Potential difference and current in simple electric circuits: A study of students' concepts, Am. J. Phys. 51, 407 (1983).

[2] C. von Rhöneck, The introduction of voltage as an independent variable-The importance of preconceptions, cognitive conflict and operating rules, in Aspects of Understanding Electricity. Proceedings of an International Workshop, Ludwigsburg, 1984, edited by R. Duit, W. Jung, and C. von Rhöneck (Schmidt and Klaunig, Kiel, 1985), pp. 275-286; see also R. Cohen, Causal relations in electric circuits: students' concepts, ibid., pp. 107-113.

[3] D. Psillos, P. Koumaras, and A. Tiberghien, Voltage presented as a primary concept in an introductory teaching sequence on DC circuits, Int. J. Sci. Educ. 10, 29 (1988).

[4] L. C. McDermott and P. S. Shaffer, Research as a guide for curriculum development: An example from introductory electricity. Part I: Investigation of student understanding, Am. J. Phys. 60, 994 (1992); also see the companion article, and , Research as a guide for curriculum development: An example from introductory electricity. Part II: Design of instructional strategies, 60, 1003 (1992).

[5] A. S. Rosenthal and C. Henderson, Teaching about circuits at the introductory level: An emphasis on potential difference, Am. J. Phys. 74, 324 (2006).

[6] P. V. Engelhardt and R. J. Beichner, Students' understanding of direct current resistive electrical circuits, Am. J. Phys. 72, 98 (2003).

[7] C. von Rhöneck, Student conceptions of the electric circuit before physics instruction, in Proceedings of the
International Workshop on Problems Concerning Students' Representation of Physics and Chemistry Knowledge, edited by W. Jung, H. Pfundt, and C. von Rhöneck (Pädagogische Hochschule, Ludwigsburg, 1981), pp. 194-213.

[8] See, e.g., D. M. Shipstone, A study of children's understanding of electricity in simple DC circuits, Int. J. Sci. Educ. 6, 185 (1984).

[9] L.C. McDermott and the Physics Education Group, Physics by Inquiry (Wiley, New York, 1996), pp. 383-517.

[10] A. Tiberghien, Critical review of research concerning the meaning of electric circuits for students aged 8 to 20 years, in Research on Education. Proceedings of the First International Workshops, La Londe les Maures, 1983 (Editions du CNRS, Paris, 1984), pp. 109-123.

[11] H. Schwedes, Teaching with analogies, in Proceedings of the Second PhD Summer School on European Research in Science Education, edited by D. Psillos (Art of Text SA, Thessaloniki, 1995); see also H. Schwedes and W. G. Dudeck, Teaching electricity by help of water, in Research in Science Education in Europe, edited by G. Welford, J. Osborne, and P. Scott (Falmers Press, London, 1996), pp. 50-63.

[12] We define potential with respect to a point within the circuit, not with respect to infinity as is the case in most electromagnetism problems.

[13] L.E. Woodman, Teaching Kirchhoff's laws, Am. Phys. Teacher 2, 161 (1934); see also R. A. Sawyer, Teaching Kirchhoff's laws, Am. Phys. Teacher 3, 86 (1935); 
E.J. Zimmerman, Directions for applying Kirchhoff's rules, Am. J. Phys. 25, 581 (1957).

[14] P. Licht, quoted in Ref. [3], p. 30.

[15] If the batteries are treated as ideal with exactly zero resistance, the current can split among two parallel batteries in any proportion. For real identical batteries with low but nonzero resistance, the current would split in half. We have found that typically the resistance of two batteries that appear identical is sufficiently different that differences of as much as $20 \%$ may occur. 